\title{
Miradas OLVIDADAS: LA DOCENCIA EN ChiHuAHUA EN LOS INICIOS DEL SIGLO XX
}

FERNANDO SANDOVAL

\section{RESUMEN}

$\square 1$ documento presenta un acercamiento a las condiciones de trabajo y a la vida cotidiana de los profesores en Chihuahua en la primera década del siglo XX. El texto se construyó con material acopiado en el Archivo de Concentración oficial. Se plantea la visión de que los profesores vivían condiciones específicas de trabajo asociadas al desarrollo de la última época del Porfiriato y a la gestación del movimiento revolucionario. Finalmente se destaca el valor que los expedientes de servicio -en especial el material fotográfico- tienen para el estudio de la historia de la educación.

Palabras clave: Historia de la práctica docente, vida cotidiana escolar, historia de la educación

SUMMARY 
The paper presents an approach to working conditions and daily life of teachers in elementary schools in Chihuahua in the first decade of the twentieth century. The text was constructed with materials stockpiled in the Archivo de Concentración of the State Government. The teachers in this period lived specific working conditions associated with the development of the last era of Porfirio Díaz presidency in the entity, and with the gestation of the revolutionary movement. Finally, the value of the records -especially the photographic material- for the study of the history of education stands out.

Keywords: History of teaching practice, everyday life in schools, history of education

\section{PRESENTACIÓN}

El gobierno del estado de Chihuahua conserva un amplio registro documental de la operación de sus diversas dependencias en su Archivo de Concentración. Ahí se almacenan, en un apartado especial, los expedientes de los profesores y profesoras chihuahuenses desde 1880 hasta finales del siglo pasado en miles de legajos que conservan las historias profesionales de quienes ejercieron la docencia al servicio de la administración estatal. Se trata de un recurso de valor incalculable para la historia de la educación en la entidad, porque constituye una fuente primaria a las vidas cotidianas de las y los docentes en un periodo histórico clave para el estado de Chihuahua. Adicionalmente, se trata de documentos que nos permiten aproximarnos a la vivencia de la práctica docente en aquellos años desde un mirador íntimo y desagregado, dado el carácter personal de los expedientes. Se trata de colecciones documentales que narran cómo fue que cada profesor asumió la profesión, cuál fue su salario durante el ejercicio de la práctica docente, qué enfermedades padeció, cuáles fueron sus desafíos y sus logros... cada evento de importancia en la vida personal y profesional de estas personas se registró en su expediente, que guarda aún constancia de esta dimensión de la vida de las escuelas públicas en Chihuahua. 
Los expedientes ofrecen un contenido adicional de gran valor: muchos de ellos contienen fotografías originales de los profesores, incluidas por razones administrativas en diversos documentos oficiales, especialmente en las hojas de servicio que se acopiaban periódicamente. Revisarlos permite conocer literalmente cara a cara a los protagonistas de las historias que aparecen al leer los antiguos oficios y cartas.

En las páginas siguientes se ofrece una visión de diversas dimensiones de lo cotidiano para estos maestros y maestras en Chihuahua antes del inicio del movimiento revolucionario de 1910. Se trata de una aproximación construida a partir de los primeros hallazgos de la exploración de los expedientes de personal, que aún aguardan una revisión exhaustiva. El Archivo de Concentración es una amplia veta para la investigación historiográfica de la práctica docente (entre otros ámbitos de la historia de la administración pública estatal), y seguramente será en el futuro material para aproximaciones nuevas al tema educativo, específicamente en lo relacionado con la vida cotidiana de las y los docentes dentro y fuera de las escuelas en las que desempeñaron su trabajo.

\section{LA VIDA EN SEPIA}

El siglo veinte empezó en el estado de Chihuahua trayendo consigo una avalancha de cambios. El primero y más importante fue una relativa vuelta a la tranquilidad perdida muchos años atrás asociada a las guerras indígenas del siglo XIX (Orozco, 1992). Durante esos primeros años del nuevo siglo Chihuahua se serenó. La entidad poco a poco se iba "civilizando" según se afirmaba en un periódico de ese entonces, y sus páginas se llenaban con chabacanas noticias del robo de una bicicleta, o de la pérdida de un par de medias de seda de uno de los almacenes de la ciudad (El Correo de Chihuahua, 1906). La prueba máxima de ese alejamiento de la barbarie lo constituían los nuevos adelantos tecnológicos: primero el tendido de las vías de ferrocarril a Juárez y a la ciudad de México en 1882, dos años después el teléfono y por esas mismas fechas la luz eléctrica asombraron los ojos de los chihuahuenses (Lloyd, 1987). Poco después llegarían hasta Chihua- 
hua las máquinas de coser Singer, los primeros automóviles y aparatos eléctricos de aire acondicionado (Lloyd, 2001).

Los progresos del estado se reflejaban en su capital. La ciudad de Chihuahua se había convertido en una urbe que rondaba los sesenta mil habitantes, marcada por el refinamiento arquitectónico de algunos de sus edificios; a la vieja catedral con sus adornos barrocos ahora le hacían compañía numerosas edificaciones nuevas: el Instituto Científico y Literario, enfrentado al Palacio de Gobierno y con la plaza Hidalgo en medio; el Hospital Porfirio Díaz y el flamante Teatro de los Héroes, con sus enormes paredes coloradas (Instituto Nacional de Estadística y Geografía, 2009). El desarrollo de la capital de Chihuahua se reflejó en su desdoblamiento espacial por medio de la fundación de nuevas colonias: por esas fechas se pusieron los cimientos de las primeras casas en el Santo Niño, en el barrio del Pacífico y en el rumbo de la Penitenciaría. En 1910, el erudito José María Ponce de León propuso comprender la anatomía de la ciudad usando como herramienta la figura de un pentágono: la base del dibujo correspondía al río Chuvíscar, uno de sus bordes laterales se formaba desde la junta de los ríos, donde estaba la estación del tren, hasta el Cerro Coronel, y el otro desde el Santuario de Guadalupe hasta el Cerro Grande. El último lado se formaba entre los dos cerros. Por ahí, escribió elegante Ponce de León, "se asoma la ciudad, como muchacha coqueta” (Ponce de León, 1907).

El crecimiento de los asentamientos humanos afectó profundamente la vida cotidiana. Por ejemplo, el uso de los tiempos libres se modificó de manera drástica en el transcurso de apenas diez años: entre 1900 y 1910 Chihuahua conoció el cinematógrafo, el fonógrafo y diversos ritmos musicales norteamericanos y europeos (Balderrama Montes y Pérez Galindo, 1999). Los cambios en la vida de todos los días se complementaron con la llegada de muchos productos nuevos. Por la vía del tren comenzaron a llegar a Chihuahua alimentos enlatados, refrescos, revistas y libros, géneros multicolores, joyas de fantasía, relojes y otros artículos (Lau, 1992). También en tren llegaron al estado hombres y mujeres de muchas latitudes, atraídos por las posibilidades de trabajo: 
entre 1890 y 1910 se fundaron o reabrieron cincuenta y cuatro minerales en el estado de Chihuahua (Canudas Sandoval, 2005) que junto con las grandes propiedades agropecuarias y la industria maderera serrana se convirtieron en polos de atracción de inmigrantes. En ese marco, la actividad educativa en las escuelas chihuahuenses se presentaba pujante. A pesar de las contradicciones sociales y económicas presentes en el estado y que originarían el estallido del movimiento revolucionario en Chihuahua en 1910, en los salones de clases de la entidad se presentaban procesos bien interesantes y complejos de desarrollo educativo.

\section{LOS NUEVOS PROFESORES}

En 1905 Rómulo Escobar, uno de los dos célebres hermanos que en ese año fundaron la Escuela de Agricultura en Ciudad Juárez, recomendó a los lectores del periódico que editaba:

Saquen ustedes un maestro de escuela de cualquier parte, al azar, pepénenlo del montón, sin escogerlo; cualquiera es igual a los otros y pónganselo en la palma de la mano. Fíjense bien en él. Está flaco el pobre, triste y meditabundo, con el brillo de la vista apagado y la dispepsia en el estómago, tiene que gastar él solo toda la bilis que deberíamos gastar los padres de los chamacos (Escobar \& García García, 2005).

Además de sus problemas biliosos, los maestros y maestras chihuahuenses de los primeros años del siglo XX tenían que lidiar con otros conflictos. El primer obstáculo era el de convertirse en profesor. Como un efecto de la profesionalización del servicio educativo, las "casas de amigas", establecimientos de educación informal instaladas en domicilios particulares en donde se repasaba el silabario de San Miguel, tendieron a desaparecer (Coromias, 2014). El avance de la institucionalización del servicio educativo y la asunción del gobierno como instancia monopolizante de la oferta educativa generaron un proceso de normalización del acceso al servicio docente. 
De todas maneras, para aquel que decidiera aventurarse por los caminos de la enseñanza, estaba bien claro que no le esperaba un futuro de holganza económica: nadie se hacía rico enseñando. Al contrario, los profesores con frecuencia se veían en la necesidad de emplearse en otras actividades alejadas de la docencia para poder completar el gasto familiar. En esta época inició también entre los maestros la práctica de endeudarse a cuenta de salarios futuros para obtener de los comerciantes artículos y servicios de diversa naturaleza (Gobierno del Estado de Chihuahua, 1906). Es llamativo que en los expedientes de algunos maestros chihuahuenses de finales del siglo XIX todavía se pueden leer antiguos pagarés por la entrega de la leche semanal, o por un juego de sala, junto con las reiteradas notas de los tenderos para que los profesores pagaran (Gobierno del Estado de Chihuahua, 1906). Estamos hablando además de una época en la que los profesores de Chihuahua no se habían organizado como gremio, por lo que no contaban con alternativas de financiamiento o préstamos de ningún tipo además de las que ofrecían las instituciones bancarias instaladas en el estado por esas fechas. Estas dimensiones de la práctica docente y de la construcción de la vida cotidiana dentro y fuera de la escuela están aún inexploradas. Se trata de un ámbito de estudio particularmente interesante, porque se relaciona con la forma en la que la identidad gremial del magisterio chihuahuense fue construyéndose en esos primeros años del siglo XX.

\section{LA JORNADA ESCOLAR}

Aunque en la dimensión nacional no existía una reglamentación clara que normara los procesos educativos a finales del siglo XIX, la rutina escolar tendía a grandes rasgos hacia la homogeneidad (Larios Guzmán y Hernández Orozco, 2012). Ello, en el marco de salones de clase no siempre adecuados a las necesidades de los educandos. Dice Ignacio Manuel Altamirano en "Beatriz", uno de sus de sus cuentos inconclusos, que algunos de los salones en los que trabajaban los alumnos eran magníficos, pues se hallaban modelados según los aposentos que se destinaban a los criminales en las cárceles de aquel tiempo (Altamirano, 1970). 
Luego de un primer lapso de estudio, generalmente se concedía a los estudiantes entre quince minutos y media hora de recreo, en que los niños jugaban a la pelota, a los huesos de chabacano, al trompo o bien a "el diablo y la monja", para luego continuar con la jornada escolar, que normalmente se volvía a interrumpir cerca de las doce del día para la comida. Lo común era que las actividades escolares matutinas se complementaran con jornadas por la tarde de dos o tres horas diarias (Gobierno del Estado de Chihuahua, 1906). Los tiempos destinados al recreo en muchas escuelas se vivían separando a los niños por sexo, de forma que se contaba con patios de juego separados para niños y niñas.

En muchos casos el profesor hacía funciones eminentemente punitivas de las conductas presuntamente inmorales o libertinas de sus alumnos. Un sufrido exalumno de la época recordaba en su adultez que:

...en la cátedra, un profesor lleno de sabiduría nos explicaba el texto y nos ponía de rodillas de cuando en cuando, si no sabíamos la clase, o bien nos hacía encerrar en un cuarto un rato, o nos pegaba en el trasero con una varita de membrillo. En otras escuelas el castigo era todavía más enérgico, se aplicaban al alumno vago, cualesquiera que fuera su edad, sendos golpes con una palmeta (Gobierno del Estado de Chihuahua, 1906).

En muchos casos el precepto de "la letra con sangre entra" se aplicaba. El uso de la palmeta para castigar a quien observaba mala conducta o no demostraba aplicación, o el empleo de las agujas o el dedal para las clases de bordado, que en ocasiones servía para corregir la falta de talento o disciplina de las menores, o los métodos pedagógicos que se basaban en la memorización y no en la comprensión, así como los castigos físicos combinados de los profesores y los padres de familia, fueron el eje de la educación moral y religiosa durante gran parte del siglo XIX y de principios del XX en Chihuahua y en muchas otras latitudes (Robles, 1997). Este énfasis de los profesores en la aplicación de sanciones diversas formaba parte importante del currículo oculto, y estaba en concordancia con las propuestas curriculares de la época, que 
se cimentaban en la idea de que la disciplina era la madre del aprendizaje efectivo y la base del carácter y los buenos modales. También tenían que ver con la conformación del rol social del profesor al interior de la sociedad septentrional, como un proveedor de conocimientos pero también de promotor de un conjunto de valores prescritos para los niños y socialmente legitimados por los chihuahuenses (Robles, 1997).

El aprendizaje de la lectura y la escritura estaban en primer lugar de prioridades. Se enseñaba a leer, escribir y las cuatro reglas de cuentas. Algunas escuelas agregaban clases de dibujo y de manualidades para las niñas, aunque este plan de estudios sufría modificaciones bien amplias de acuerdo a los tiempos, a la formación de los profesores y a los libros de texto que se encontraban a la mano. Frecuentemente se hacía hincapié en la formación valoral de los niños, casi siempre mediante el empleo de fábulas como recursos didácticos (Gobierno del Estado de Chihuahua, 1906).

Resulta interesante revisar cómo estas escuelas chihuahuenses de principios del siglo pasado presentaban rasgos profundamente distintos a lo que ocurre hoy en día en los establecimientos escolares de nuestra entidad, y al mismo tiempo estaban ya ahí otros elementos que subsisten hoy en día. Es posible presenciar así cómo las escuelas chihuahuenses públicas del siglo XXI abrevan en esta emergencia temprana del sistema educativo estatal en los años previos al inicio del conflicto armado de 1910.

\section{LAS ESCUELAS}

Los planteles escolares de principios del siglo XX estaban marcados por la heterogeneidad. La necesidad sentida por muchos padres de familia en el sentido de que sus hijos tuvieran la oportunidad de contar con una formación escolar generó la creación o adaptación de numerosos espacios que se consagraron a las actividades educativas (Gobierno del Estado de Chihuahua, 1906). En muchos casos se trataba tan solo de edificaciones originalmente pensadas como casas habitación que se acondicionaban para recibir a los niños y a sus maestros; en otros casos, los menos, las escuelas eran planeadas y construidas por arquitectos especia- 
lizados, con base en las últimas prescripciones higiénicas y pedagógicas de la época. En medio de estos dos extremos podían encontrarse escuelas instaladas en las más variadas condiciones: casas de hospedaje acondicionadas como centros, anexos de edificios públicos, bodegas; prácticamente cualquier lugar que pudiera albergar a un grupo de niños y a su profesor era bueno. Las áreas de juego eran amplias y se procuraba que en las escuelas en las que se ofrecía servicio a niños y niñas estuvieran divididas por sexo. Estos espacios servían también para la realización de diversas actividades cívicas. Las canchas deportivas hicieron su aparición en las escuelas de Chihuahua poco después.

El arquetipo de esta manera de entender los centros educativos fue materializado en 1906, con la construcción de la escuela no. 141, en la esquina de la avenida Penitenciaría (hoy 20 de Noviembre) y Terrazas de la ciudad de Chihuahua. La construcción original de este plantel reflejaba las nociones que se consideraban ideales para la práctica educativa y sirvió de modelo a proyectos arquitectónicos escolares posteriores: se trataba de un edificio central, compuesto por un salón de actos con altos techos y pisos de madera, y a su alrededor corredores que daban acceso a las aulas; todo ello rodeado por dos áreas de juego (Sandoval Gutiérrez, 2003). Una excepción en la austeridad reinante en el equipamiento de las escuelas la constituían los centros educativos de la Sociedad Filomática, instalados también en la ciudad de Chihuahua. Estos planteles fueron fundados por grupos de padres de familia pudientes con el propósito de asegurar que sus hijos pudieran vivenciar procesos educativos de alta calidad. Estas escuelas fueron equipadas trayendo de El Paso, Texas, lo último en menaje y material escolar (Sociedad Filomática de Chihuahua, México, 1908).

\section{LOS NIÑOS Y LAS NIŃAS}

Del otro lado de la moneda educativa aparecían los niños chihuahuenses. A pesar de que el paradigma escolar dominante en ese entonces promovía la imagen del profesor como el protagonista de lo que ocurría al interior de los salones de clase, entonces 
como hoy, en realidad los verdaderos personajes centrales de la realidad educativa eran los niños y las niñas que llenaban los salones de clase. Estos chicos fueron espectadores a la vez que protagonistas de la avalancha de transformaciones en la cultura y en la vida cotidiana chihuahuense de los años previos a la Revolución, y vivieron esta realidad en el contexto de las escuelas y de sus salones de clase.

Ser niño en el estado de Chihuahua a principios del siglo pasado implicaba vivir la infancia en un mundo que ofrecía amplios espacios para relacionarse con la naturaleza, para aprender a partir de la experiencia, pero que también implicaba el enfrentamiento con rígidas posiciones de la mayor parte de los adultos con respecto a cómo deberían educarse los infantes.

Parte de esta rigidez se mostraba en un interés por reconocer a los mejores. En 1906 el Gobernador Enrique Creel ordenó que de manera mensual los centros educativos reportaran a la estructura educativa los nombres de los alumnos con mejores desempeños para integrar una lista de honor que se publicaba en la gaceta oficial. Esta medida generó pugnas bien intensas por ocupar los lugares de privilegio en esa lista, que significaba la exhibición pública de los méritos académicos de los chicos (Mayer, 2007).

Los niños tenían otras alternativas para alcanzar la atención de la gente. Eran especialmente apreciados por la comunidad chihuahuense los cuadros gimnásticos, que se practicaban con indumentarias especiales y que consistían en la ejecución bien precisa de diversos movimientos y desplazamientos por parte de grupos numerosos de alumnos (Gobierno del Estado de Chihuahua, 1906). Estas exhibiciones se preparaban para algún evento especial, la conmemoración de alguna fecha histórica de importancia o con motivo de la visita de un personaje ilustre. Los cuadros gimnásticos se inspiraban en obras literarias protagonizadas por duendecillos o hadas mágicas, o por dragones de hocico llameante y figuras rutilantes, y eran acompañados con frecuencia con piezas de oratoria, disciplina que era cultivada con ahínco en muchas primarias y que se consideraba como una habilidad muy importante (Gobierno del Estado de Chihuahua, 1906). Todas estas expresiones del arte desplegado por los niños chihuahuen- 
ses se vieron dilatadas en toda su magnitud en la visita del Presidente Porfirio Díaz al estado, apenas unos pocos meses antes del estallido de la revolución. Para esa fecha se organizó en la capital de la "Fiesta de la niñez chihuahuense", que fue celebrada en el Teatro de los Héroes. Ahí Díaz disfrutó de los alcances artísticos de los alumnos y alumnas de las escuelas primarias de la ciudad (Ayuntamiento de Chihuahua, 1993).

Para los alumnos menos afectos al movimiento corporal y al ejercicio, existía una opción más: la participación en cuadros estáticos que consistían en composiciones vivientes acerca de un tema específico: las fuerzas naturales, una escena histórica, algún tópico que se pretendía explicar mediante su representación visual, una especie de performance temprano que casi siempre era fotografiado para la posteridad. Estos cuadros estáticos servían adicionalmente para hacer públicas las habilidades para las artes manuales de los docentes, que se encargaban de la confección de sus materiales.

En realidad, la escuela dejaba poco tiempo libre a los niños. La disposición discontinua de los horarios de clase mantenía a los alumnos en los planteles educativos mucho tiempo más que a los niños de la actualidad, ocupados en actividades de muy diversa índole. Más allá de las puertas de la escuela quedaba un mundo por explorar. Por otro lado, no eran pocos los niños y niñas del campo y la ciudad que complementaban su actividad escolar con el trabajo, generalmente en auxilio de las actividades de los padres. Esto era especialmente frecuente en el campo, sobre todo en las temporadas en las que los ciclos agrícolas exigían el trabajo de cuantas manos estuvieran disponibles. El concepto del trabajo infantil como una práctica reprochable no llegaría a Chihuahua hasta muchos años después.

Estamos hablando además de una época en la que la distinción asociada al género como un procedimiento selectivo para decidir quién podía ir a la escuela y quién se quedaba en casa o en el trabajo era mucho más importante que hoy en día. En las estadísticas de inscripción de las escuelas de la época casi invariablemente el número de niños supera al de las niñas, en una 
tendencia que iba a mantenerse por décadas (Gobierno del Estado de Chihuahua, 1906).

\section{LOS MATERIALES PARA ENSEŃAR}

Desde los años finales del siglo XIX las profesoras y profesores chihuahuenses tenían a su disposición diversos tipos de textos: cuadernos de lecciones, que contenían unidades subtemáticas pequeñas que podían abordarse en una clase; libros de ejercicios, con cuestionarios y otras actividades; libros de lectura y textos de consulta (Gobierno del Estado de Chihuahua, 1906).

Los cuadernos de lecciones ofrecían temas, frecuentemente numerados, estructurados en pequeños módulos de una o dos páginas que podían estudiarse durante una jornada. Típicamente, la lección contenida en estos libros empezaba con una definición de sus conceptos clave para luego revisar algunos significados de nociones complementarias del tema principal. En la mayor parte de los cuadernos de lecciones se recomendaba que, al terminar de revisar el texto, se pasara al cuaderno de actividades o a los ejercicios en el pizarrón, de ahí que las editoriales que surtían a los profesores chihuahuenses de la época acompañaran los libros de lecciones con abundantes cuadernos de ejercicios, que se vendían en paquete (Gobierno del Estado de Chihuahua, 1906).

Estos materiales se complementaban con textos de consulta. Este tipo de libros se utilizaba frecuentemente como referencia para la planeación de las lecciones de clase que el profesor dictaría a sus alumnos, y ocasionalmente los mismos niños tenían acceso a estos libros para buscar información o bien para repasar los contenidos abordados en clase. Además, había textos que eran de uso cotidiano en las escuelas: los "alfabetos" (científico, de artes y oficios, de juegos de la infancia, de juegos de la juventud, alfabeto mitológico, alfabeto zoológico), colecciones de fichas de cartoncillo sobre diversos tópicos; el "Amigo de los niños", escrito en francés por Sabatie y traducido en 1880 por Escoiquiz; la "Aritmética” de Navea; una amplia variedad de catecismos (histórico, de Ripalda, de economía doméstica, de geografía universal, de urbanidad, de perseverancia) y un buen número de compendios: 
de los derechos, de la urbanidad, etcétera, todos ellos junto a los textos para la enseñanza de la moral y las buenas costumbres, como el "Manual del buen tono" o el "Manual de urbanidad y de buenas maneras" de Urcullu (Benso Calvo, 1997).

En la ciudad de Chihuahua, los profesores podían conseguir estos materiales en la librería y papelería "Pluma y lápiz", propiedad de Eugenio de la Torre. Otro recurso era pedirlos por correo a la ciudad de México, en donde se editaban e imprimían la mayoría de los materiales de apoyo para profesores en casas libreras como "Herrero hermanos sucesores", "Librería de la viuda de Ch. Bouret", “Tipografía de Aguilar e hijos” y otras (Castañeda Rincón, 2006).

Ocasionalmente, los libros contenían, además de las actividades de ejercitación de las habilidades memorísticas, ejercicios llamados ideológicos, que consistían en preguntas que motivaban procesos reflexivos en los alumnos. Otro clásico de la época era "El lector católico mexicano", que contenía narraciones acompañadas de viñetas en blanco y negro -salvo la ilustración de la portada, que era en todos los colores- que iban hilvanando una historia con conceptos de biología, religión o historia. Los contenidos de las lecciones no se agrupaban bajo ningún criterio, de manera tal que cada tomo de "El lector católico" era una sabrosa ensalada temática. Algunas lecciones incluían al final preguntas sobre su contenido, bajo el nombre de "ejercicios de lenguaje", y en otras más se recomendaba al profesor narrar a sus alumnos el final de una fábula, o bien pedirles que lo hicieran ellos mismos (Cadena, 1903).

\section{LA REALIZACIÓN PERSONAL}

Surgida en condiciones complejas, la profesión magisterial en el estado de Chihuahua suponía la asunción de diversos supuestos. Uno de ellos era su potencial para que las personas que decidían convertirse en profesores o en maestras pudieran alcanzar un grado de realización personal que desde luego tenía que ver primariamente con el tema del sustento, pero que también operaba en otros niveles. De entrada, los profesores y las maestras recibían tratamientos especiales por parte de la sociedad chihuahuense 
de entonces; las maestras eran tratadas de "señoritas", mientras que los profesores siempre eran llamados "maestros" (Gobierno del Estado de Chihuahua, 1906). Estos formulismos revelan por sí mismos elementos simbólicos bien poderosos asociados a la autoridad, el respeto, la sabiduría. Ser profesor implicaba tener un punto de vista informado, ser considerado por la comunidad como un miembro importante, estratégico en más de un sentido.

La profesión también traía consigo una imagen que resultaba gratificante para muchos. El canon de la formalidad actuaba como un regulador de la figura de los profesores hacia la sociedad pero también como un elemento simbólico de autoafirmación para los docentes.

Otra dimensión de la realización personal tenía que ver con el apostolado magisterial incipiente que ya se gestaba por aquellos años. Los profesores eran los mensajeros del progreso, los fundadores de la civilización, los portadores de la luz del conocimiento, y su influencia educativa y cultural fue determinante para el derrotero de los destinos de nuestro estado. En la vida de muchas localidades rurales del estado hicieron su aparición las kermeses, las fiestas cívicas y las graduaciones; la vida cotidiana de las localidades que contaban con un profesor se modificó sustancialmente, al grado que en algunos lugares las fiestas de la escuela desplazaron a las celebraciones tradicionales (Gobierno del Estado de Chihuahua, 1906).

La importancia que los profesores tenían para la sociedad chihuahuense era apoyada por la postura de las autoridades estatales al respecto. En los primeros años del siglo XX fueron varios los profesores que recibieron medallas y diplomas de reconocimiento al mérito académico y laboral por iniciativa del propio gobernador del estado (Gobierno del Estado de Chihuahua, 1906).

Si el reconocimiento público de la labor de los maestros era considerable, y la valoración que se hacía de su labor era muy alta, no es posible decir lo mismo de sus emolumentos. Una lista de compras de un profesor de la ciudad de Chihuahua que se encuentra en un expediente de 1909 incluye los siguientes productos: harina, chile, cominos, una caja de sal, una veladora, un frasco de vinagre, almidón, un bote de polvo para limpiar trastos, 
una caja de jabón y un paquete de cigarros (Gobierno del Estado de Chihuahua, 1906). En otro sentido, es interesante observar cómo, infortunadamente, los primeros años del surgimiento de lo que sería el sistema educativo estatal también son los años de la emergencia de problemas serios: en varias partes de Chihuahua aparecen casos de profesores con serios problemas de alcoholismo, tal vez asociados a las condiciones de soledad y marginación en que muchos de ellos trabajaban. Aparecen también problemas de salud relacionados con cuadros neuróticos, estrés, problemas en la garganta y otros malestares (Gobierno del Estado de Chihuahua, 1906). Había otras dificultades. No existía un servicio médico establecido para los trabajadores de la educación y los gastos relacionados con la atención de la salud de los maestros corrían por su cuenta. Los medicamentos eran un gasto aparte.

\section{EL FINAL DE LA JORNADA}

Los profesores chihuahuenses de principios del siglo XX transitaban por el ejercicio de la docencia con diversos ritmos y con destinos mucho más inciertos que los de los profesores de la actualidad. No había límites cronológicos para ejercer como profesor o profesora. Los docentes no tenían una edad establecida para jubilarse o pensionarse porque no se había implementado en ese entonces ningún servicio de pensiones, y aunque generalmente el gobierno del estado atendía los gastos de pensión de los profesores, esta disposición estaba sujeta al arbitrio de los administradores (Gobierno del Estado de Chihuahua, 1906).

La comparación de la serie de fotografías de un mismo profesor, que inicia con la imagen adjunta a la hoja de ingreso al servicio docente y se cierra con la fotografía pegada a la carta testamentaria, permiten atisbar en alguna medida los abismos profundos de su personalidad y las peculiaridades de la Chihuahua que le tocó enfrentar. Es una verdadera ventana al pasado a través de los ojos de un maestro. Tener la oportunidad de ver a los ojos a estos maestros y maestras que actuaron en la Chihuahua de hace cien años es un verdadero privilegio. Nos permite acercarnos un poco a su vida de todos los días y comprender me- 
jor la magnificencia de sus esfuerzos personales y colectivos para construir un mundo mejor a través de su labor profesional. Las fotografías y expedientes de los profesores mostrados plantean adicionalmente otros retos relacionados con la calidad del servicio educativo que se ofrece en las escuelas de nuestra entidad hoy. ¿Habrán mejorado los servicios educativos en un siglo? ¿Qué opinarían al respecto los maestros y las maestras que aparecen en este trabajo?

\section{TRABAJOS Y DOCUMENTOS CITADOS}

Altamirano, I. (1970). Clemencia, cuentos de invierno, Julia, Antonio, Beatriz, Atenea. México: Porrúa.

Álvarez, M. (1960). La educación en Chihuahua: estudio histórico para las bodas de oro de la Normal de Chihuahua. México.

Ayuntamiento de Chihuahua (1993). Visita a Chihuahua del señor presidente general don Porfirio Díaz. Octubre de 1909. Chihuahua: Ayuntamiento de Chihuahua.

Balderrama Montes, R., \& Pérez Galindo, R. (1999). La música en Chihuahua. 1890-1940. Chihuahua: Universidad Autónoma de Chihuahua.

Benso Calvo, C. (1997). Controlar y distinguir: la enseñanza de la urbanidad en las escuelas del siglo XIX. Vigo: Servicio de Publicacións da Universidade de Vigo.

Cadena, L. (1903). El lector católico mexicano: método de lectura conforme con la inteligencia de los niños : libro segundo, basado en las lecciones de cosas, en la religión católica, en la sana moral : y los rigurosos cánones preceptuados por la moderna ciencia educativa. Herrero Hermanos.

Canudas Sandoval, E. (2005). Las venas de plata en la historia de México. México: Universidad Juárez Autónoma de Tabasco.

Castañeda Rincón, J. (2006). La enseñanza de la geografía en México. Una visión histórica. 1821-2005. México: Plaza y Valdés.

Coromias, F. (2014). Educar hoy. Madrid: Ediciones Palabra.

Dale Lloyd, J. (1987). El proceso de modernización capitalista en el noroeste de Chihuahua (1880-1910). México: Universidad Iberoamericana.

Dale Lloyd, J. (2001). Cinco ensayos de cultura material de rancheros y medieros del noroeste de Chihuahua, 1886-1910. México: Universidad Iberoamericana. 
El Correo de Chihuahua (1906, octubre 8). Se roban una bicicleta. El Correo de Chihuahua, p. 2.

Escobar, R., \& García García, J. (2005). Don Rómulo Escobar; artículos y ensayos (1896-1946). Ciudad Juárez: Municipio de Juárez.

Gobierno del Estado de Chihuahua (1906). Archivo de Concentración de la Administración Estatal. Fondo expedientes de personal de educación. Chihuahua: Gobierno del Estado de Chihuahua.

Instituto Nacional de Estadística y Geografía (2009). Estadísticas históricas de México. México: INEGI.

Larios Guzmán, M., \& Hernández Orozco, G. (2012). Desempeño y profesionalización: las maestras de párvulos en la historia de la educación en Chihuahua, México, durante las primeras décadas del siglo XX. Historia Caribe, 147-157.

Lau, R. (1992). Historia general de Chihuahua (vol. 3). Ciudad Juárez: Universidad Autónoma de Ciudad Juárez.

Mayer, A. (2007). México en tres momentos: 1810-1910-2010. Hacia la conmemoración del Bicentenario de la Independencia y del Centenario de la Revolución Mexicana. Retos y perspectivas. México: Universidad Nacional Autónoma de México.

Orozco, V. (1992). Las guerras indias en la historia de Chihuahua. Primeras fases. México: Consejo Nacional para la Cultura y las Artes.

Ponce de León, J. (1907). Datos geográficos y estadísticos del estado de Chihuahua. Chihuahua: Imprenta del Gobierno.

Robles, M. (1997). Educación y sociedad en la historia de México. México: Siglo XXI Editores.

Sandoval Gutiérrez, F. (2003). La escuela modelo, microhistoria de un centro escolar. Chihuahua: Instituto Chihuahuense de la Cultura.

Sociedad Filomática de Chihuahua, México (1908). Escuelas incorporadas para páruulos, niños y niñas. Chihuahua: Taller de "El Norte". 


\section{Anexo fotográfico}

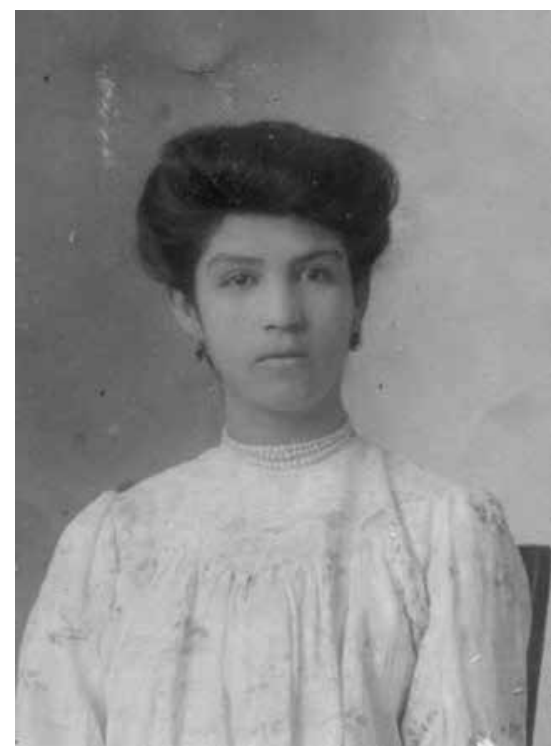

Imagen 1. Profesora Ana Acosta. Archivo de Concentración.

Fondo de personal magisterial.

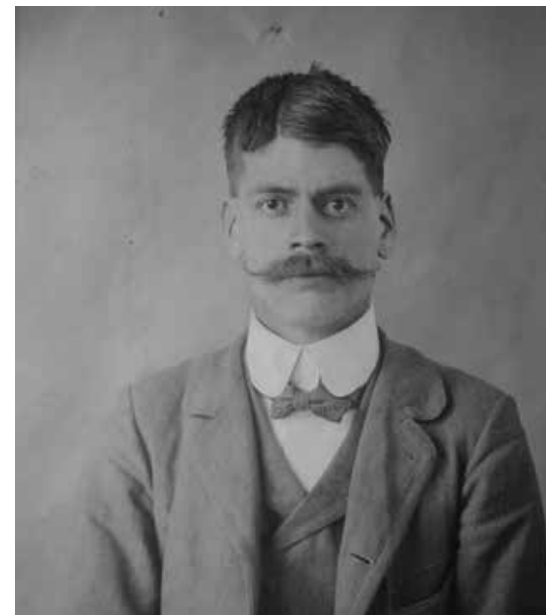

Imagen 3. Profesor sin identificar. Archivo de Concentración.

Fondo de personal magisterial.

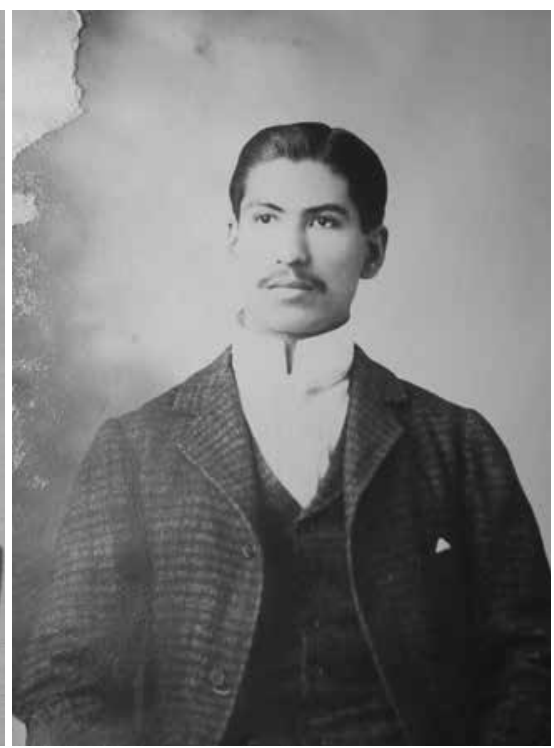

Imagen 2. Profesor sin identificar. Archivo de Concentración. Fondo de personal magisterial.

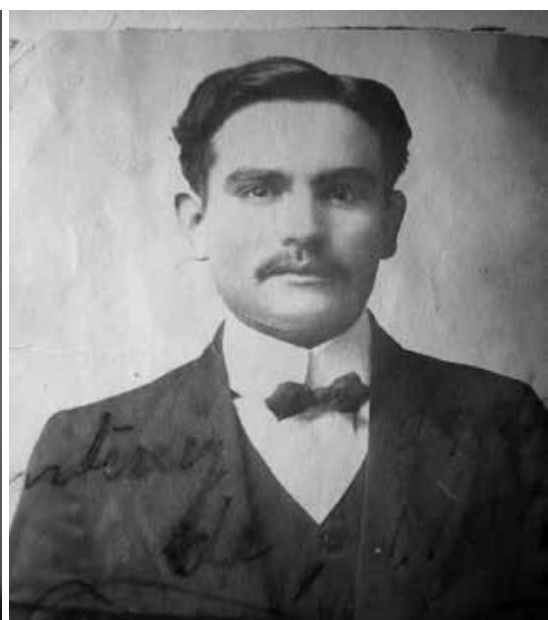

Imagen 4. Profesor Tomás Sáenz. Archivo de Concentración. Fondo de personal magisterial. 


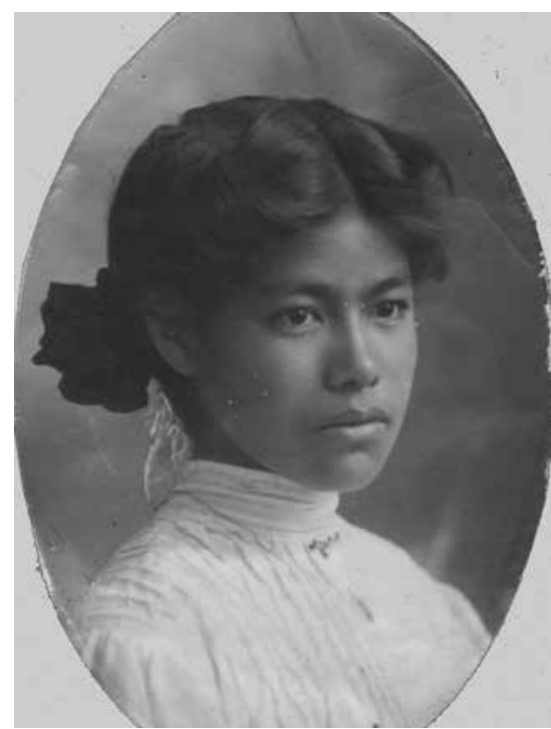

Imagen 5. Profesora sin identificar. Archivo de Concentración. Fondo de personal magisterial.

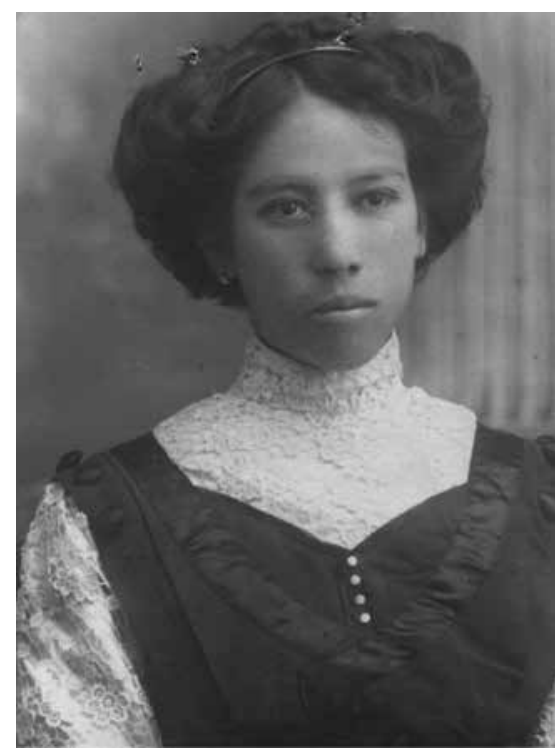

Imagen 7. Profesora sin identificar. Archivo de Concentración. Fondo de personal magisterial.

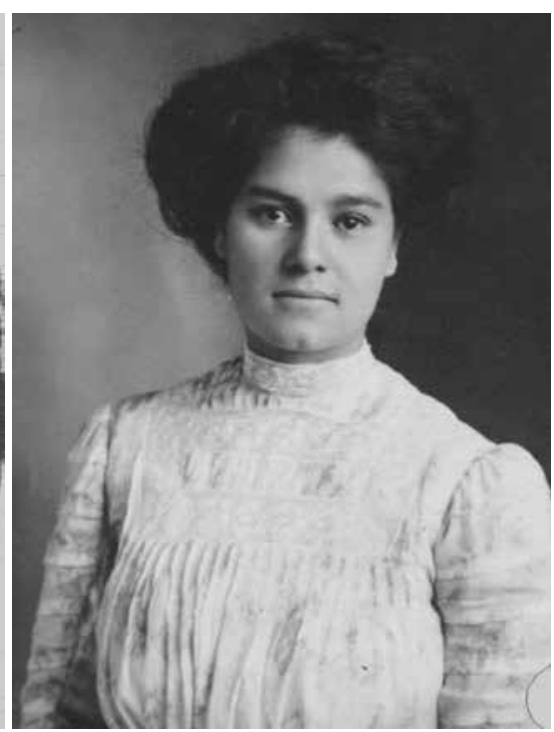

Imagen 6. Profesora sin identificar. Archivo de Concentración. Fondo de personal magisterial.

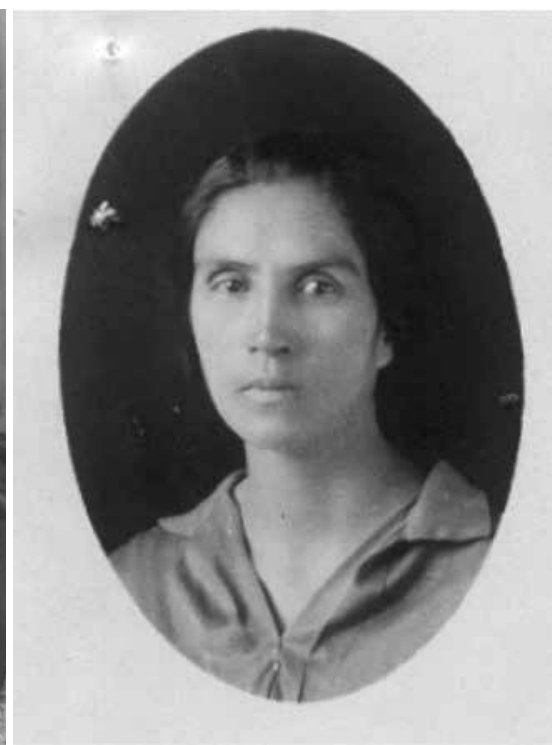

Imagen 8. Ana Acosta de Mendoza, 37 años de edad, profesora de quinta clase con sueldo de 60 pesos. Archivo de Concentración. Fondo de personal magisterial. 


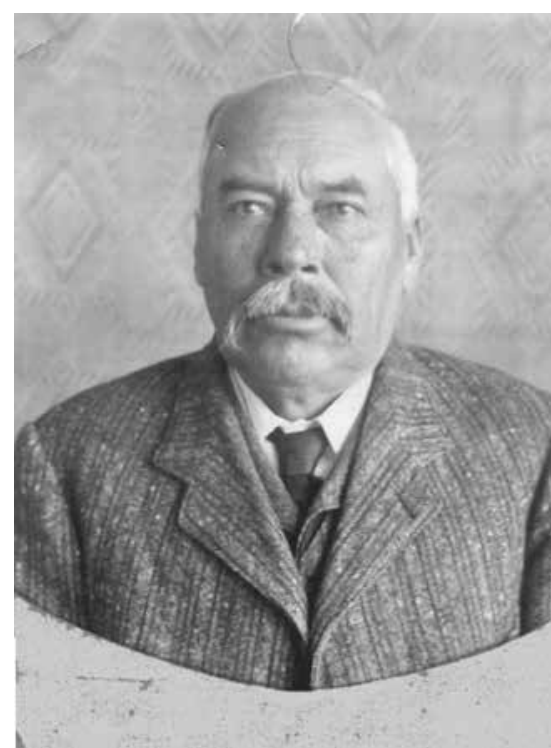

Imagen 9. Profesor Carlos Ahumada, escuela 84 de Santo Tomás. Archivo de Concentración. Fondo de personal magisterial.

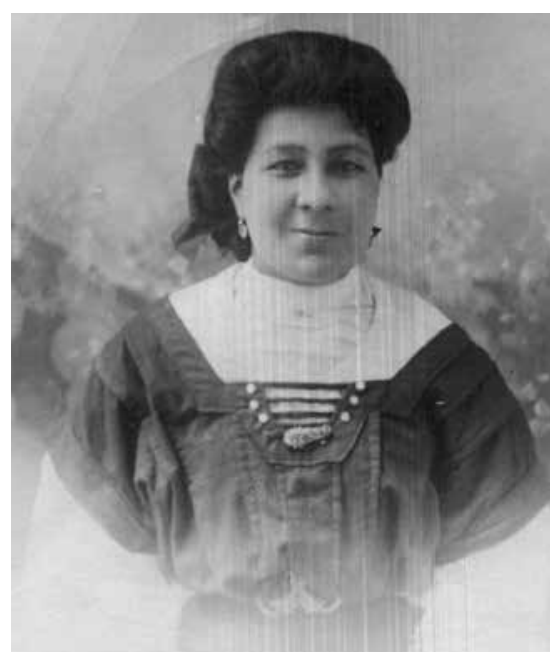

Imagen 11. Profesora Cayetana Almanza, Escuela 212 de Ocampo, mayo 23 de 1909. Archivo de Concentración. Fondo de personal magisterial.

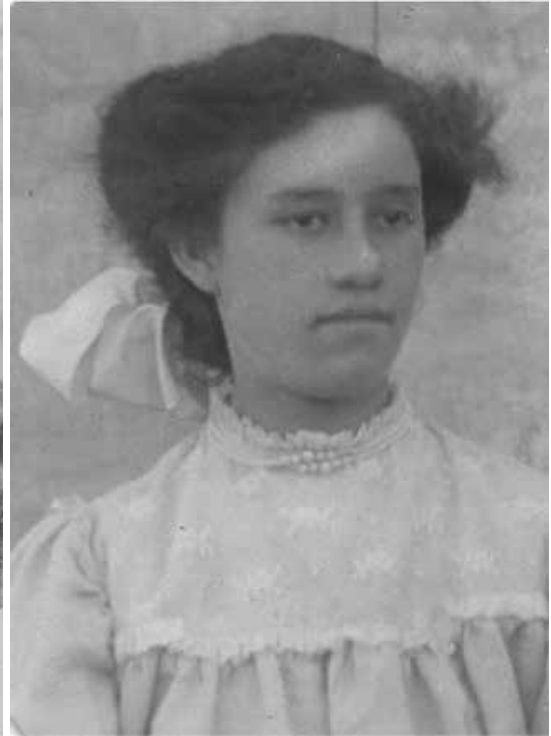

Imagen 10. Profesora Carmen Alvarado, escuela 33 de Villa Ahumada, agosto de 1909. Archivo de Concentración.

Fondo de personal magisterial.

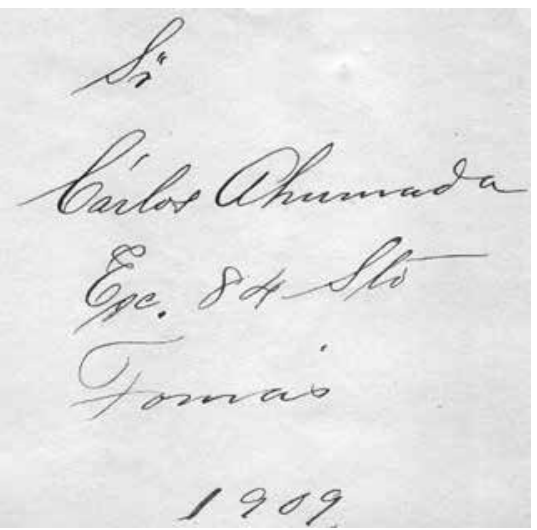

Imagen 12. Reverso de imagen, profesor Carlos Ahumada, escuela 84 de Santo Tomás, 1909. Archivo de Concentración. Fondo de personal magisterial. 


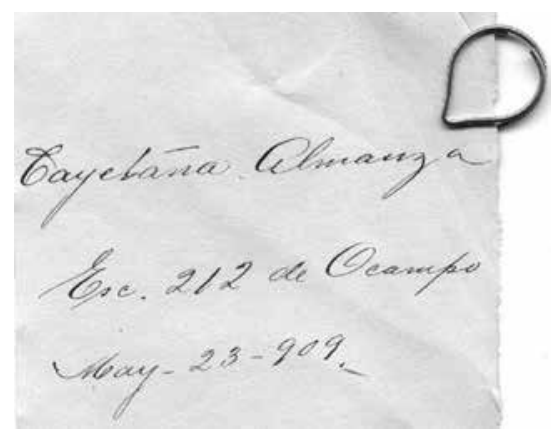

Imagen 13. Reverso de imagen, profesora Cayetana Almanza, Escuela 212 de Ocampo, mayo 23 de 1909. Archivo de Concentración. Fondo de personal magisterial.

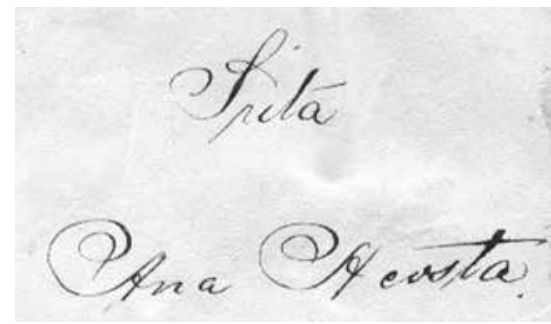

Imagen 15. Reverso de imagen, Señorita Ana Acosta. Archivo de Concentración. Fondo de personal magisterial.

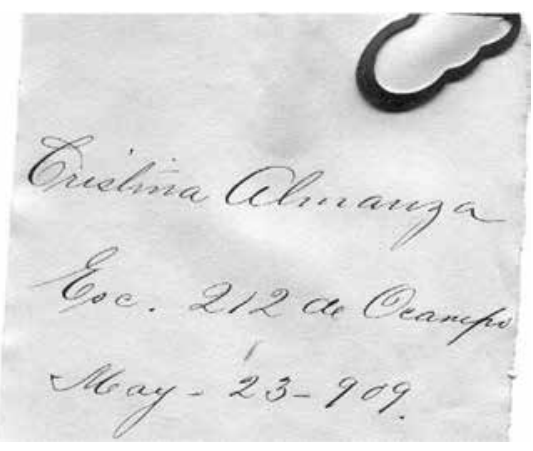

Imagen 14. Reverso de imagen, profesora Cristina Almanza, Escuela 212 de Ocampo, mayo 23 de 1909. Archivo de Concentración. Fondo de personal magisterial.

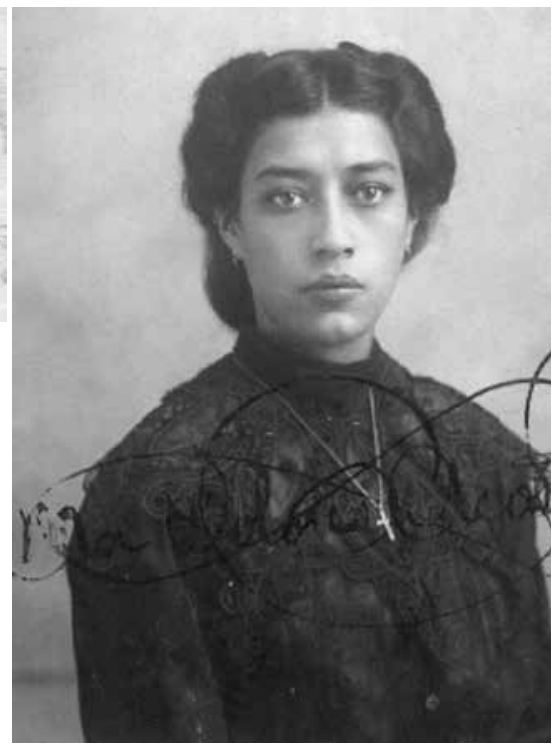

Imagen 16. Profesora María Dolores Acosta, 1910 (hoja de ingreso al servicio docente). Archivo de Concentración. Fondo de personal magisterial. 


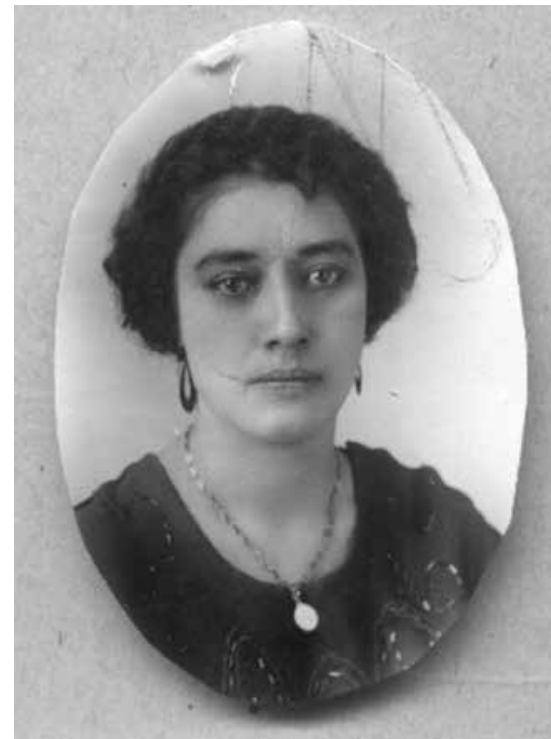

Imagen 17. Profesora María Dolores Acosta, 1923. Archivo de Concentración. Fondo de personal magisterial.

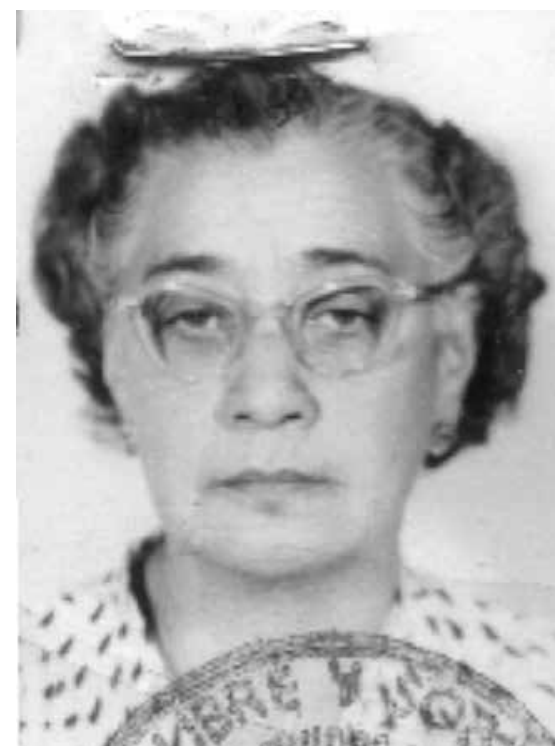

Imagen 19. Profesora María Dolores Acosta, 1961. Archivo de Concentración. Fondo de personal magisterial.

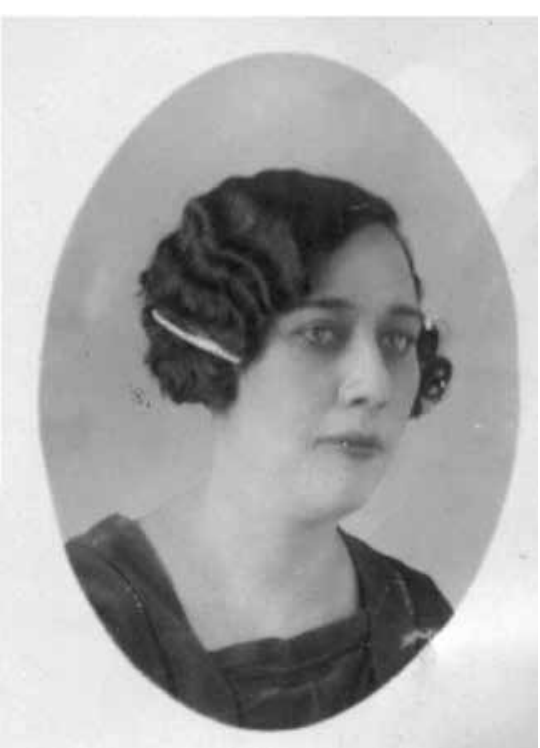

Imagen 18. Profesora María Dolores Acosta, 1930. Archivo de Concentración. Fondo de personal magisterial.

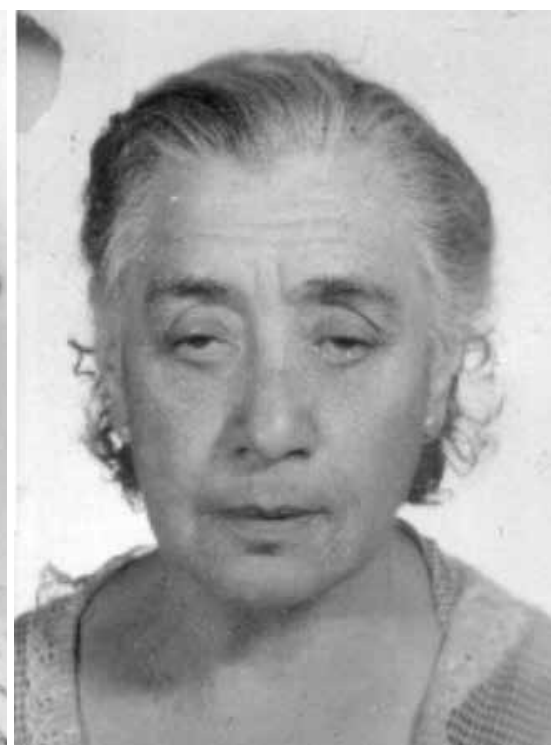

Imagen 20. Profesora María Dolores Acosta, 1962. Archivo de Concentración. Fondo de personal magisterial. 
MiRADAS OLVIDADAS: LA DOCENCIA EN CHIHUAHUA EN LOS INICIOS DEL SIGLO XX

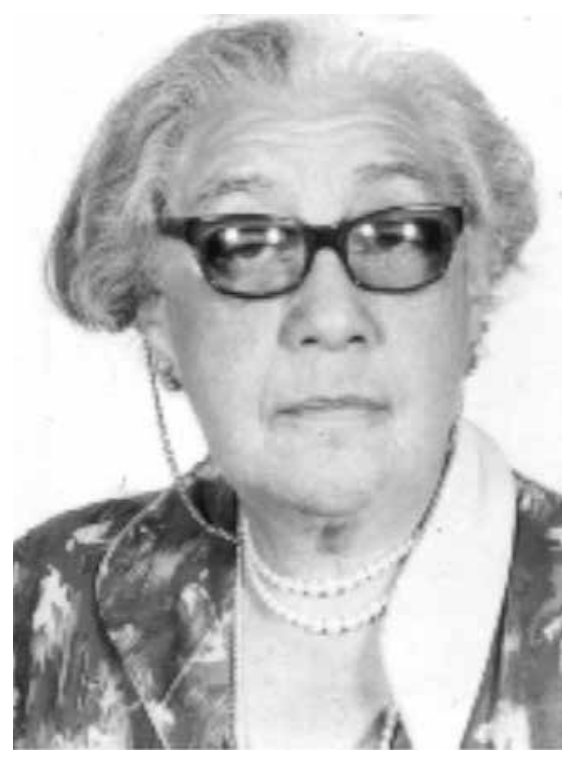

Imagen 21. Profesora María Dolores Acosta, 1971 (carta testamentaria). Archivo de Concentración. Fondo de personal magisterial. 